\title{
Index of sources and main topics (first citation)
}

Allison, G. T., 155

Alvesson, M., 5

Amason, A. C., 139

Arendt, H., 16

Armstrong, P., 198

Armstrong, P., 95

Asch, S. E., 142

Babcock, D. L., 3

Baron, J., 55

Bastide, S., 91

Bazerman, M. H., 68

Beach, L. R., 126

Beck, U., 91

Berg, M., 39

Bloor, G., 22

Bolman, L. G., 29

Bord, R. J., 91

Brehmer, B., 66

Brown, J., 41

Brunswik, E., 102

Burris, B., 47

Buss, D. M., 91

Callon, M., 34

Clegg, S. R., 24 cognitive processes

decision and action, 108

goals, 101

holistic decision process, 99

influences on cue selection and use, 109

influences on quasi-rational judgement, 112

problem or opportunity recognition, 102

thought and judgement, 102

Cohen, M. D., 123

Coleman, G., 204

Collins, R., 41

Cooksey, R. W., 78

Cox, R. A., 87

Craib, I., 44

Crespi, F., 16

Cyert, R. M., 27

Dawes, A., 21

Dean, J. W., 163

decision analysis

constraints, 65

example, 59 
expected utility theory (EU), 57

managers and risk, 69

prospect theory, 67

realism, 63

risky choice, 56

usefulness, 70

Delbecq, A. L., 196

Deluca, D. R., 91

Di Maggio, 33

Donaldson, L., 28

Dryden, P. S., 87

Ducot, C., 202

Eden, C., 198

Eiser, J. R., 181

Ellis, D. G., 139

engineering managers

decision implications, 50

history, 39

merging roles, 47

positivism, 38

professionals, 41

European Federation, 86

Fast, J. C., 73

Fayol, H., 25

Fischer, F., 39

Fredrickson, J. W., 162

French, S., 63

Galbraith, J. R., 26

Giddens, A., 16

Glasbergen, P., 176

Glover, I. A., 41

Goodwin, P., 16

group decisions

case study, 145

conforming groups, 142

constraints, 141

groupthink, 143 management, 144

myths and reality, 135

polarized groups, 143

team decisions, 139

Hales, C. P., 5

Hammond, K. R., 78

Harrison, F. E., 164

Hartley, J., 44

Hickson, D., 158

Hill, G. W., 138

Hill, S., 39

Hirokawa, R. Y., 138

Hofstede, G., 8

Hogarth, R. M., 65

Hwang, C., 76

Hynes, M., 113

individual decisions

case study, 132

constraints on reasoned choice, 119

decision management, 129

decisions make cowards, 125

experimental decisions, 123

first choices, 121

goals, 122

image theory, 126

information, 121

obligations, 125

random decisions, 123

reasoned choice, 118

recognition-primed decisions, 128

symbolic decisions, 124

Isenberg, D. J., 8

Jaccard, J., 9

Jackall, R., 125

Jamous, H., 42 
Janis, I. L., 118

Kahneman, D., 67

Kasperson, R. E., 93

Kelly, G. A., 198

Kipnis, D., 13

Kleindorfer, P. R., 137

Klein, G. A., 128

Kline, T. J. B., 107

Korsgaard, M. A., 139

Kriger, M. P., 160

Kumar, K., 39

Kunreuther, H., 92

Larson, M. S., 45

Latour, B., 34

Law, J., 22

Lichtenstein, S., 113

Lindblom, C. E., 120

Linstone, H. A., 157

Lloyd, B. U., 40

Lukes, S., 16

Luthans, F. R., 7

management communication, 10

decision implications, 19

influence tactics, 13

power, 15

purpose, 3

thinking, 8

unwritten rules, 15

work, 5

March, J. G., 11

Mayer, J. P., 25

McCall, M. W., 160

Mintzberg, H., 5

Mitchell, T. R., 12

Molloy, S., 203

Montgomery, H., 122
Moore, P. G., 63

Morgan, M. G., 16

Moscovici, S., 142

Mullen, J. D., 65

multiattribute choice

AHP, 76

policy capturing, 78

SMART, 74

without probabilities, 80

NATO ASI, 86

Nohria, N., 33

Nutt, P. C., 117

Orasanu, J., 139

organizations

actor-networks, 34

administration mode of ordering, 23

decision implications, 35

enterprise mode of ordering, 30

networks, 33

points of view, 20

Otway, H. J., 91

Parkin, J. V., 78

Parnaby, J., 45

Parsons, T., 16

Payne, J. W., 108

Penrose, E., 3

Peters, T. J., 29

Pfeffer, J., 32

Philipson, L. L., 86

Pitblado, R. M., 89

Plous, S., 67

Raelin, J., 44

Reader, W. J., 42

Reason, J., 90

risk analysis

amplification, 93 


\section{INDEX}

cultures, 90

managing, 95

probabilistic, 86

psychology, 91

stigma, 94

technical construction, 85

Robson, M., 196

Rowe, A. J., 116

Saaty, T. L., 76

Schoemaker, P. J. H., 202

Silverman, D., 21

Simon, H. A., 63

Sinclair, A., 136

Slovic, P., 92

Stallen, P. J. M., 92

Stohl, C., 12

Stoner, J. A. F., 144

strategic decisions effective processes, 164

Mintzberg et al. model, 165 multiple perspectives, 155

organization structure and context, 162

power model, 167

problem type and complexity,

158

strategic decision management

case study, 187

concluding principles, 184

translating the problem, 173

translating the solution, 180 strategic management tools analysis of interests, 195

anticipating cue sets, 195

brainstorming, 196

case study, 207

cognitive maps, 198

delphi, 198

good argument, 205

information technology, 203

logical framework, 204

nominal group technique, 196

scenarios, 199

value trees, 199

Sundstrom, E., 137

Thomas, K. W., 32

Thompson, E. P., 40

Toulmin, S. E., 180

Trainer, F. E., 111

Tversky, A., 107

von Winterfeldt, 57

Vroom, V. H., 136

Wartofsky, M. W., 91

Watson, S. R., 45

Weber, M., 24

Whitley, R., 3

Whittaker, J., 90

Willmott, H., 21

Wright, G. N., 57

Zey, M., 118 